\title{
The microbiological profile and presence of bloodstream infection influence mortality rates in necrotizing fasciitis
}

\author{
I-Chuan Chen ${ }^{1,3}$, Wen-Cheng Li, ${ }^{3,4}$, Yu-Cheng Hong ${ }^{1,3}$, Shian-Sen Shie ${ }^{2,3}$, Wen-Chih Fann ${ }^{1,3}$ and \\ Cheng-Ting Hsiao ${ }^{1,3^{*}}$
}

\begin{abstract}
Introduction: Necrotizing fasciitis (NF) is a life threatening infectious disease with a high mortality rate. We carried out a microbiological characterization of the causative pathogens. We investigated the correlation of mortality in NF with bloodstream infection and with the presence of co-morbidities.

Methods: In this retrospective study, we analyzed 323 patients who presented with necrotizing fasciitis at two different institutions. Bloodstream infection (BSI) was defined as a positive blood culture result. The patients were categorized as survivors and non-survivors. Eleven clinically important variables which were statistically significant by univariate analysis were selected for multivariate regression analysis and a stepwise logistic regression model was developed to determine the association between BSI and mortality.

Results: Univariate logistic regression analysis showed that patients with hypotension, heart disease, liver disease, presence of Vibrio spp. in wound cultures, presence of fungus in wound cultures, and presence of Streptococcus group A, Aeromonas spp. or Vibrio spp. in blood cultures, had a significantly higher risk of in-hospital mortality. Our multivariate logistic regression analysis showed a higher risk of mortality in patients with pre-existing conditions like hypotension, heart disease, and liver disease. Multivariate logistic regression analysis also showed that presence of Vibrio spp in wound cultures, and presence of Streptococcus Group A in blood cultures were associated with a high risk of mortality while debridement $>=3$ was associated with improved survival.
\end{abstract}

Conclusions: Mortality in patients with necrotizing fasciitis was significantly associated with the presence of Vibrio in wound cultures and Streptococcus group A in blood cultures.

\section{Introduction}

Understanding the mechanisms underlying the pathophysiology of an infectious disease and characterization of the causative organism are key to providing better medical or surgical care, preventing complications and initiating early, appropriate antimicrobial treatment. Necrotizing fasciitis (NF), a life-threatening infectious disease with mortality rate ranging from $17 \%$ to $34 \%$ [1-6] actually refers to a spectrum of diseases where necrosis of deeper soft tissue is driven by an infective microorganism [7]. NF primarily involves the superficial

\footnotetext{
* Correspondence: giomacky@gmail.com

'Department of Emergency Medicine, Chang Gung Memorial Hospital, No.6,

W. Sec., Jiapu Rd., Puzih City, Chiayi County 613, Taiwan Full list of author information is available at the end of the article
}

fascia with extensive deterioration of the surrounding tissue. It has been suggested that the rapid, soft tissue necrosis seen in NF is caused by the release of bacterial toxins and enzymes, which may then lead to extensive inflammation, sepsis and multiple organ failure [8]. We previously developed a laboratory risk indicator for necrotizing fasciitis (LRINEC), which is a useful tool to distinguish necrotizing soft tissue infections from other soft tissue infections [9].

The overall annual incidence of necrotizing fasciitis in the USA was reported as $0.04 / 1,000$ people [10]. NF has been classified based on different criteria such as the anatomical level of involvement or the requirement of surgical management [11]. However, it is most convenient to categorize NF based on the microbiological characteristics of
C Biomed Central 
the pathogen involved [12-14]. Type 1 necrotizing fasciitis is a polymicrobial infection arising from aerobic and anaerobic bacteria, while Type 2 necrotizing fasciitis is caused by group A Streptococcus with or without a coexisting Staphylococcal infection. Although necrotizing fasciitis caused by fungi was previously classified under Type 2, fungal necrotizing fasciitis has recently been classified under its own category [15].

Although early diagnosis of NF is key to managing the disease, it is complicated by the fact that NF typically presents with vague, non-specific symptoms. Treatment typically consists of a combination of surgical debridement, antibiotic treatment based on the pathogen and oxygenation of the injured tissue [16]. Immunocompromise, diabetes mellitus, alcoholism, end-stage renal disease, malignancy and chemotherapy have all been suggested as predisposing factors in NF [16]. However, NF can also occur in otherwise healthy adults and is usually precipitated by some form of trauma $[17,18]$.

A number of studies have looked at prognostic factors in NF. Diabetes mellitus was shown to be significantly associated with mortality $[16,19]$. Our previous study of 128 patients showed that Vibrio infection and Aeromonas infection were strongly associated with mortality [20]. Mortality rates in NF are also influenced by the presence of bloodstream infection (BSI) $[6,12]$. However, the impact of individual, causative bacteria in BSI and outcomes has not yet been studied in patients with necrotizing fasciitis.

The primary endpoint of the present study was to characterize the causative microorganism of necrotizing fasciitis. The secondary endpoint was to investigate the correlation between mortality and the causative pathogens of a wound or blood stream infection.

\section{Materials and methods}

This retrospective study enrolled a total of 323 consecutively presenting, necrotizing fasciitis patients who were admitted between January 2002 and September 2005 via the emergency department (ED) of two different hospitals (the Gueishan Chang Gung Medical Center and the Chiayi Chang Gung Memorial Hospital). Out of a total of 354 necrotizing fasciitis patients, 31 patients were excluded from the study due to a lack of records. The study sites included a tertiary academic center, serving a population of approximately one million, with an annual ED census of 180,000 visits per year and a community, university-affiliated hospital, serving a population of approximately 0.3 million, with an annual ED census of 60,000 visits per year. Both hospitals had 24 hour inhouse coverage by a senior plastic or orthopedic surgery resident, with an attending plastic or orthopedic surgeon available within 30 minutes.
Inclusion criteria were 1) patients who had undergone surgery and 2) availability of histology and pathology reports. A diagnosis of necrotizing fasciitis is usually difficult and cannot be based solely on microbiological findings. In this study, a diagnosis of necrotizing fasciitis was based on clinical manifestations and surgical findings in our hospital and was confirmed by either clinical, microbiologic, radiographic, gross anatomic findings or histologic examination. Information collected at the time of admission included age, gender, comorbidities, clinical symptoms/signs, site and etiology of infection, emergency department (ED) triage vital signs, laboratory findings at the time of admission and medication being taken at the time of admission. The time from admission to operation, the need for amputation, the duration of hospitalization, and the mortality rates were reviewed and the microbiology of wound culture and blood culture were also documented. The study population was very heterogenous in order to ensure the inclusion of a large number of causative pathogens. Hypotension was defined as a systolic blood pressure $<90 \mathrm{mmHg}$ at ED triage. Positive wound or blood culture was defined as successful culture from the wound or blood within three days. Infection was defined as the presence of a pathogenic microorganism in a wound culture or blood culture or as a clinically suspected infection plus the administration of antibiotics [21,22]. Sepsis was defined by the guidelines of the American College of Chest Physicians/Society of Critical Care Medicine Consensus Conference as an infection plus two systemic inflammatory response syndrome criteria $[21,22]$. Bloodstream infection (BSI) was defined as a positive growth of bacteria or fungus from at least one blood culture. Following hospital protocol, all patients were administered antibiotics prior to surgery. Once NF was suspected, patients were immediately administered third generation cephalosporin (Ceftriaxone) with metronidazole or clindamycin at the emergency department. Vancomycin was only prescribed at the emergency department if methicillin-resistant Staphylococcus aureus (MRSA)-related NF was suspected. The number of debridements was based on the clinical judgment of the surgeon.

All wound cultures obtained aseptically during the initial surgery, were placed in standard aerobic and anaerobic swab and transport tubes and plated within one hour in our laboratory on standard sheep blood agar plates. Chocolate, casein-soy and MacConkey agar media were used for aerobic bacteria and standard anaerobic medium and broth were used for anaerobic bacteria. Plates were incubated at $36^{\circ} \mathrm{C}$ under aerobic or anaerobic conditions. None of the patients received IVIG or Xigris treatment. The study was approved by the Institute Reviewing Board of Chang Gung Memorial Hospital and 
informed consents from patients were waived. Laboratory standard protocol for the bacterial culture was followed.

\section{Statistical analysis}

Demographic data were expressed as the mean with standard deviation for numerical data, and $\mathrm{n}$ with percentage (\%) for contingency data. Microbiologic characteristics were summarized as $\mathrm{n}(\%)$ for 1 ) subjects who were positive for wound culture growth, 2) subjects positive for blood culture growth (single etiologic agent) and 3) subjects positive for blood culture growth (multiple etiologic agents). We used the Pearson chi-square test or Fisher's exact test to compare the dispersion in microbiologic characteristics between subjects with single etiologic agent, and those with multiple etiologic agents. We also used Fisher's exact test to compare the association of patients' mortality with microbiologic characteristics. In addition, we performed univariate and multivariate logistic regression analyses to identify the prognostic factors which might be associated with mortality. A $P$-value less than 0.05 was considered statistically significant. All statistical analyses were performed using SPSS 15.0 statistics software (SPSS Inc, Chicago, IL, USA).

\section{Results}

A total of 323 patients with necrotizing fasciitis were enrolled in this study. Table 1 shows the demographic characteristics of the patients. Males comprised $69.3 \%$ of the study population (224 males) and the average age was 57.5 years $(\mathrm{SD}=15.3)$. The mortality rate was $16.1 \%(52 /$ $323)$. We observed that $279(86.4 \%)$ subjects were positive for growth of wound cultures, and 65 (20.1\%) subjects were positive for growth of blood cultures (Table 1). Sixty percent of our study population was diabetic and $68.7 \%$ of the patients underwent surgery within 24 hours of admission.

Table 2 summarizes the microbiologic characteristics of the 279 patients who were positive for growth of wound cultures. Of the 279 patients, there were 126 patients with a single etiologic agent, and 153 patients with multiple etiologic agents. In the single etiology group, we found a significantly higher dispersion of subjects with Methicillin-susceptible S. aureus (MSSA) when compared with the multiple etiology group. Vibrio species, methicillin-resistant Staphylococcus aureus (MRSA), group A beta-hemolytic Streptococcus and Klebsiella pneumoniae were other the most common pathogens in the single etiology group.

The multiple etiology group showed a significantly higher number of patients with Gram positive cocci such as Enterococcus fecalis and S. viridians and Gram negative rods such as E. coli., Enterobacter spp., Acinetobacter baumannii, and Pseudomonas aeroginosa when compared with the single etiology group.
Table 1 Demographic characteristics of 323 patients with necrotizing fasciitis

\begin{tabular}{|c|c|}
\hline Demographic characteristics & $(\mathrm{N}=323)$ \\
\hline $\mathrm{Age}^{1}$, years & $57.5 \pm 15.3$ \\
\hline Gender $^{2}$, male (\%) & $224(69.3)$ \\
\hline \multicolumn{2}{|l|}{ Pre-existing disease $^{2}$} \\
\hline Diabetes mellitus & $195(60.4)$ \\
\hline Liver disease & $91(28.2)$ \\
\hline Renal disease & $60(18.6)$ \\
\hline Heart disease & $43(13.3)$ \\
\hline Peripheral vascular disease & $27(8.4)$ \\
\hline Intravenous drug use & $15(4.6)$ \\
\hline \multicolumn{2}{|l|}{ Clinical features ${ }^{2}$} \\
\hline Infected site, limbs & $276(85.4)$ \\
\hline Tender & $261(80.8)$ \\
\hline Erythema & $252(78.0)$ \\
\hline Presence of sepsis & $244(75.5)$ \\
\hline Tachycardia & $152(47.1)$ \\
\hline Bullae formation & $132(40.9)$ \\
\hline Fever & $103(31.9)$ \\
\hline Hypotension & $42(13.0)$ \\
\hline \multicolumn{2}{|l|}{ Therapeutic factors and the outcomes } \\
\hline Operation within 24 hours $^{2}$ & $222(68.7)$ \\
\hline Amputation $^{2}$ & $83(25.7)$ \\
\hline Mortality $^{2}$ & $52(16.1)$ \\
\hline Number of debridement ${ }^{1}$, number & $3.14 \pm 2.2$ \\
\hline Hospital length of stay ${ }^{1}$, days & $35.0 \pm 26.1$ \\
\hline Wound culture, positive (\%) & $279(86.4)$ \\
\hline Blood culture, positive (\%) & $65(20.1)$ \\
\hline
\end{tabular}

1,2 Data were expressed as mean \pm SD for ${ }^{1}$ numerical data, and $\mathrm{n}(\%)$ for ${ }^{2}$ contingency data.

The microbiologic characteristics of 65 patients who were positive for growth of blood cultures are summarized in Table 3. Of the 65 patients, there were 61 patients with a single etiologic agent, and 4 patients with multiple etiological agents. In the single etiology group, we found a significantly lower number of patients with $E$. coli. when compared with the multiple etiology group, while Vibrio species, MSSA and MRSA were the most common pathogens in this group.

We investigated the association of mortality with microbiological profile in the 279 patients who were positive for growth of wound cultures (Table 4) and in the 65 patients who were positive for growth of blood cultures (Table 5). Of the 38 patients with Gram negative bacterial BSI in our study, $81.6 \%$ had sepsis and $50 \%$ had hypotension at ER triage (data not shown). We showed that mortality was significantly associated with the presence of Clostridium spp. $(P=0.035)$ and Vibrio spp. $(P<0.001)$ in wound cultures.

We used univariate and multivariate logistic regression analyses to look at the relationship between mortality and the presence of specific microorganisms in wound 
Table 2 Microbiologic characteristics of 279 patients who had positive growth of wound cultures

\begin{tabular}{|c|c|c|c|}
\hline Variable & $\begin{array}{l}\text { Single etiologic agent } \\
(n=126)\end{array}$ & $\begin{array}{l}\text { Multiple etiologic agents } \\
\qquad(n=153)\end{array}$ & $P$-value \\
\hline \multicolumn{4}{|l|}{ Gram-positive cocci } \\
\hline \multicolumn{4}{|l|}{ Aerobic } \\
\hline \multicolumn{4}{|l|}{ Staphylococcus aureus } \\
\hline Methicillin-susceptible S. aureus & $27(21.4)$ & $15(9.8)$ & $0.007^{*}$ \\
\hline Methicillin-resistant S. aureus & $20(15.9)$ & $34(22.2)$ & 0.182 \\
\hline Coagulase-negative Staphylococcus & $2(1.6)$ & $11(7.2)$ & $0.042^{*}$ \\
\hline \multicolumn{4}{|l|}{ Streptococcus } \\
\hline Beta-hemolytic (Group A) & $13(10.3)$ & $24(15.7)$ & 0.188 \\
\hline Beta-hemolytic (Group B) & $2(1.6)$ & $4(2.6)$ & 0.693 \\
\hline Non-group A or B & - & $2(0.7)$ & - \\
\hline S. viridans & $2(1.6)$ & $36(23.5)$ & $<0.001^{*}$ \\
\hline Enterococcus faecalis & $1(0.8)$ & $45(29.4)$ & $<0.001^{*}$ \\
\hline \multicolumn{4}{|l|}{ Anaerobic } \\
\hline Peptostreptococcus & - & $38(13.6)$ & - \\
\hline \multicolumn{4}{|l|}{ Gram-positive rods } \\
\hline \multicolumn{4}{|l|}{ Aerobic } \\
\hline Corynebacterium & - & $8(2.9)$ & - \\
\hline \multicolumn{4}{|l|}{ Anaerobic } \\
\hline Clostridium spp. & - & $5(1.8)$ & - \\
\hline \multicolumn{4}{|l|}{ Gram negative rods } \\
\hline \multicolumn{4}{|l|}{ Aerobic } \\
\hline Acinetobacter baumannii & $3(2.4)$ & $20(13.1)$ & $0.001^{*}$ \\
\hline Aeromonas spp. & $11(8.7)$ & $11(7.2)$ & 0.635 \\
\hline Citrobacter spp. & $2(1.6)$ & $9(5.9)$ & 0.119 \\
\hline E.coli. & $2(1.6)$ & $35(22.9)$ & $<0.001^{*}$ \\
\hline Enterobacter spp. & $1(0.8)$ & $19(12.4)$ & $<0.001^{*}$ \\
\hline Klebsiella pneumoniae & $13(10.3)$ & $28(18.3)$ & 0.061 \\
\hline Pseudomonas aeruginosa & $3(2.4)$ & $16(10.5)$ & $0.008^{*}$ \\
\hline Vibrio spp. & $21(17.5)$ & - & - \\
\hline Eikenella corrodens & - & $4(2.6)$ & - \\
\hline Klebsiella oxytoca & - & $3(2.0)$ & - \\
\hline Proteus mirabillis & - & $23(15.0)$ & - \\
\hline Serratia marcescens & - & $7(4.6)$ & - \\
\hline Other gram negative rods & $1(0.8)$ & $15(9.2)$ & $0.002^{*}$ \\
\hline \multicolumn{4}{|l|}{ Anaerobic } \\
\hline Prevotella spp. & $2(1.6)$ & $10(6.5)$ & 0.071 \\
\hline Bacteroides fragilis & - & $15(9.8)$ & - \\
\hline Fusobacterium & - & $1(0.7)$ & - \\
\hline Veillonella & - & $3(2.0)$ & - \\
\hline Fungus & - & $6(3.9)$ & - \\
\hline
\end{tabular}

Data were summarized as $\mathrm{n}(\%)$ for the subjects with positive growth of wound culture by single etiologic agent, and multiple etiologic agent, respectively. * $P<0.05$ significantly different between single, and multiple etiologic agents, $P$-values were derived through Pearson Chi-square test, or Fisher's exact test.

and blood cultures. We also investigated the relationship of mortality with other prognostic factors (Tables 6 and $7)$. We showed a higher risk of mortality in patients with hypotension (OR: (95\% confidence interval $(\mathrm{CI}))=$ 7.07 (3.48 to 14.37$), P<0.001)$, heart disease (OR: $(95 \%$ $\mathrm{CI})=3.52(1.72$ to 7.20$), P=0.001)$, liver disease (OR. $(95 \% \mathrm{CI})=2.37$ (1.28 to 4.37$), P=0.006)$, presence of Vibrio spp. in wound cultures (OR: $(95 \% \mathrm{CI})=6.37$
(2.58 to 15.57), $P<0.001$ ), presence of fungus (candida) in wound cultures (OR: $(95 \% \mathrm{CI})=6.34$ (2.58 to 15.57 , $P<0.001)$; presence of Streptococcus group A in blood cultures (OR: $(95 \% \mathrm{CI})=8.24$ (1.34 to 50.56), $P=$ 0.023), presence of Aeromonas spp in blood cultures (OR: $(95 \% \mathrm{CI})=5.47(1.07$ to 27.89$), P=0.041)$, and presence of Vibrio spp. in blood cultures (OR: (95\% CI) $=4.95$ (1.94 to 12.64$), P=0.001$ ] (Table 6). 


\begin{tabular}{|c|c|c|c|}
\hline Variable & $\begin{array}{l}\text { Single etiologic agent } \\
(n=61)\end{array}$ & $\begin{array}{l}\text { Multiple etiologic agents } \\
\qquad(n=4)\end{array}$ & $P$-value \\
\hline \multicolumn{4}{|l|}{ Gram-positive bacteria } \\
\hline \multicolumn{4}{|l|}{ Aerobic } \\
\hline \multicolumn{4}{|l|}{ Staphylococcus aureus } \\
\hline Methicillin-susceptible S. aureus & $9(14.8)$ & $1(25.0)$ & 0.496 \\
\hline Methicillin-resistant S. aureus & $7(11.5)$ & - & - \\
\hline \multicolumn{4}{|l|}{ Streptococcus } \\
\hline Beta-hemolytic (Group A) & $5(8.2)$ & - & - \\
\hline Beta-hemolytic (Group B) & $1(1.6)$ & $1(25.0)$ & 0.120 \\
\hline \multicolumn{4}{|l|}{ Anaerobic } \\
\hline Peptostreptococcus & $1(1.6)$ & $1(25.0)$ & 0.120 \\
\hline \multicolumn{4}{|l|}{ Gram negative bacteria } \\
\hline \multicolumn{4}{|l|}{ Aerobic } \\
\hline Acinetobacter baumannii & $2(3.3)$ & - & - \\
\hline Aeromonas spp. & $6(9.8)$ & - & - \\
\hline Citrobacter spp. & $1(1.6)$ & - & - \\
\hline E.coli & $3(4.9)$ & $2(50.0)$ & $0.027^{*}$ \\
\hline Enterobacter spp. & $1(1.6)$ & $1(25.0)$ & 0.120 \\
\hline Klebsiella pneumoniae & $2(3.3)$ & $1(25.0)$ & 0.176 \\
\hline Proteus mirabillis & $1(1.6)$ & - & - \\
\hline Vibrio spp. & $20(32.8)$ & - & - \\
\hline Other gram negative rods & $1(1.6)$ & - & - \\
\hline \multicolumn{4}{|l|}{ Anaerobic } \\
\hline Prevotella spp. & $1(1.6)$ & - & - \\
\hline Bacteroides fragilis & - & $1(25.0)$ & - \\
\hline
\end{tabular}

Data were summarized as $\mathrm{n}$ (\%) for subjects with positive growth of blood culture (single etiologic agent or multiple etiologic agent).

* $P<0.05$ denotes a significant difference between single, and multiple etiologic agents, $P$-values were derived through Fisher's exact test.

Our data also showed a significantly lower risk of mortality in subjects with debridement $>=3$ times (OR: $(95 \% \mathrm{CI})=0.19$ (0.10 to 0.39), $P<0.001$ ] (Table 6).

We used multivariate logistic regression analysis to investigate the prognostic factors which were significant in the univariate logistical analysis. Since a 24-hour or more delay until the operation is a risk factor for death, the multivariate analysis was performed controlling for subjects with a 24-hour or more delay until the operation. Our data showed a higher risk of mortality in patients with pre-existing diseases, (hypotension, heart disease, and liver disease). The presence of Vibrio spp in wound cultures, and the presence of Streptococcus group A in blood cultures also increased the risk of mortality whereas patients with debridement $>=3$ times had a lower risk of mortality (Table 7).

We performed univariate analysis to investigate the association between positive blood cultures, positive wound cultures and survival (Table 8 ). We showed that patients with positive blood stream infections had a 2.26 higher incidence of death. However, we found no significant association between positive wound cultures and mortality.

\section{Discussion}

In this study, we showed that patients with necrotizing fasciitis who were positive for Vibrio spp in wound culture, or Streptococcus group A in blood culture were at higher risk for mortality. The presence of certain preexisting conditions, such as diabetes mellitus, hypotension, heart disease or liver disease, were also predictors of higher mortality, while debridement $>=3$ times was associated with a lower risk of mortality.

NF is caused by a number of different pathogens. Our study population had a predominance of polymicrobial infections $(54.8 \%, 153 / 279)$. However, our data agreed with previous studies that showed that infections caused by single organisms were not uncommon [20,23]. We found that MSSA, MRSA and Vibrio spp. were the most common pathogens in the single etiology group. Staphylococcus aureus was recently shown to be present in $18.9 \%$ of patients with necrotizing fasciitis of limbs [24] and was the most frequently $(21.7 \%)$ isolated bacteria in NF patients [11]. Wound cultures of our study patients showed 1) a predominance of Methicillin-susceptible Staphylococcus aureus, 2) a significantly lower occurrence of Gram positive cocci such as coagulase-negative 
Table 4 Association of mortality with microbiologic characteristics of $\mathbf{2 7 9}$ patients with positive growth of wound cultures

\begin{tabular}{|c|c|c|c|}
\hline Variable & $\begin{array}{l}\text { Survival } \\
(n=232)\end{array}$ & $\begin{array}{l}\text { Death } \\
(n=47)\end{array}$ & $P$-value \\
\hline \multicolumn{4}{|l|}{ Gram-positive cocci } \\
\hline \multicolumn{4}{|l|}{ Aerobic } \\
\hline \multicolumn{4}{|l|}{ Staphylococcus aureus } \\
\hline Methicillin-susceptible S. aureus & $39(16.8)$ & $3(6.4)$ & 0.075 \\
\hline Methicillin-resistant S. aureus & $47(20.3)$ & $7(14.9)$ & 0.543 \\
\hline Coagulase-negative Staphylococcus & $12(5.2)$ & $1(2.1)$ & 0.703 \\
\hline \multicolumn{4}{|l|}{ Streptococcus } \\
\hline Beta-hemolytic (Group A) & $32(13.8)$ & $5(10.6)$ & 0.645 \\
\hline Beta-hemolytic (Group B) & $4(1.7)$ & $2(4.3)$ & 0.267 \\
\hline \multicolumn{4}{|l|}{ Non-group A or B } \\
\hline S. viridans & $33(14.2)$ & $5(10.6)$ & 0.644 \\
\hline Enterococcus faecalis & $37(15.9)$ & $9(19.1)$ & 0.666 \\
\hline \multicolumn{4}{|l|}{ Anaerobic } \\
\hline Peptostreptococcus & $30(12.9)$ & $8(17.0)$ & 0.485 \\
\hline \multicolumn{4}{|l|}{ Gram-positive rods } \\
\hline \multicolumn{4}{|l|}{ Aerobic } \\
\hline Corynebacterium & $7(3.0)$ & $1(2.1)$ & 1.000 \\
\hline \multicolumn{4}{|l|}{ Anaerobic } \\
\hline Clostridium spp. & $2(0.9)$ & $3(6.4)$ & $0.035^{*}$ \\
\hline \multicolumn{4}{|l|}{ Gram negative rods } \\
\hline \multicolumn{4}{|l|}{ Aerobic } \\
\hline Acinetobacter baumannii & $20(8.6)$ & $3(6.4)$ & 0.776 \\
\hline Aeromonas spp. & $18(7.8)$ & $4(8.5)$ & 0.773 \\
\hline Citrobacter spp. & $10(4.3)$ & $1(2.1)$ & 0.697 \\
\hline E.coli. & $29(12.5)$ & $8(17.0)$ & 0.478 \\
\hline Enterobacter spp. & $15(6.5)$ & $5(10.6)$ & 0.349 \\
\hline Klebsiella pneumoniae & $36(15.5)$ & $5(10.6)$ & 0.501 \\
\hline Pseudomonas aeruginosa & $16(6.9)$ & $3(6.4)$ & 1.000 \\
\hline Vibrio spp. & $11(4.7)$ & $11(23.4)$ & $<.001^{*}$ \\
\hline Eikenella corrodens & $4(1.7)$ & - & - \\
\hline Klebsiella oxytoca & $3(1.3)$ & - & - \\
\hline Proteus mirabillis & $23(9.9)$ & - & - \\
\hline Serratia marcescens & $7(3.0)$ & - & - \\
\hline Other gram negative rods & $12(5.2)$ & $3(6.4)$ & 0.724 \\
\hline \multicolumn{4}{|l|}{ Anaerobic } \\
\hline Prevotella spp. & $12(5.2)$ & - & - \\
\hline Bacteroides fragilis & $11(4.7)$ & $4(8.5)$ & 0.292 \\
\hline Fusobacterium & $1(0.4)$ & - & - \\
\hline Veillonella & $2(0.9)$ & $1(2.1)$ & 0.426 \\
\hline Fungus & $5(2.2)$ & $1(2.1)$ & 1.000 \\
\hline
\end{tabular}

Data were summarized as $\mathrm{n}(\%)$ for subjects with positive growth of wound culture. Subjects were classified into "Death" and Survival" categories. * $P$ $<0.05$ denotes a significant difference between the Death, and Survival groups. $P$-values were derived through Fisher's exact test.

Staphylococcus, S. viridans and Enterococcus fecalis and 3) a significantly lower occurrence of Gram negative rods, such as Acinetobacter baumannii, Enterobacter spp., E. coli and Pseudomonas aeroginosa in the single
Table 5 Microbiologic characteristics of 65 patients with positive growth of blood culture by patients' mortality

\begin{tabular}{|c|c|c|c|}
\hline Variable & $\begin{array}{l}\text { Survival } \\
(n=41)\end{array}$ & $\begin{array}{c}\text { Death } \\
(n=24)\end{array}$ & $P$-value \\
\hline \multicolumn{4}{|l|}{ Gram-positive bacteria } \\
\hline \multicolumn{4}{|l|}{ Aerobic } \\
\hline \multicolumn{4}{|l|}{ Staphylococcus aureus } \\
\hline Methicillin-susceptible S. aureus & $10(24.4)$ & - & - \\
\hline Methicillin-resistant S. aureus & $5(12.2)$ & $2(8.3)$ & 1.000 \\
\hline \multicolumn{4}{|l|}{ Streptococcus } \\
\hline Beta-hemolytic (Group A) & $2(4.9)$ & $3(12.5)$ & 0.350 \\
\hline Beta-hemolytic (Group B) & $1(2.4)$ & $1(4.2)$ & 1.000 \\
\hline \multicolumn{4}{|l|}{ Anaerobic } \\
\hline Peptostreptococcus & $2(4.9)$ & - & - \\
\hline \multicolumn{4}{|l|}{ Gram negative bacteria } \\
\hline \multicolumn{4}{|l|}{ Aerobic } \\
\hline Acinetobacter baumannii & - & $2(8.3)$ & - \\
\hline Aeromonas spp. & $3(7.3)$ & $3(12.5)$ & 0.662 \\
\hline Citrobacter spp. & $1(2.4)$ & & \\
\hline E.coli & $3(7.3)$ & $2(8.3)$ & 1.000 \\
\hline Enterobacter spp. & $1(2.4)$ & $1(4.2)$ & 1.000 \\
\hline Klebsiella pneumoniae & $3(7.3)$ & & \\
\hline Proteus mirabillis & & $1(4.2)$ & \\
\hline Vibrio spp. & $11(26.8)$ & $9(37.5)$ & 0.412 \\
\hline Other gram negative rods & $1(2.4)$ & - & - \\
\hline \multicolumn{4}{|l|}{ Anaerobic } \\
\hline Prevotella spp. & $1(2.4)$ & - & - \\
\hline Bacteroides fragilis & - & $1(4.2)$ & - \\
\hline
\end{tabular}

Data were summarized as $n(\%)$ for the subjects with positive growth of blood culture by patients' mortality, respectively.

$P$-values were derived through Fisher's exact test.

etiology groups. Blood cultures showed a significantly lower occurrence of $E$. coli in the single etiology group. It would be interesting to dissect the mechanisms underlying these differences in microbiological profiles between the single etiology groups and the multiple etiology groups.

Interestingly, we showed that Vibrio spp., previously considered a rare cause of necrotizing fasciitis, accounted for 21 cases within our study population. Vibrio spp. are known to flourish in warm saltwater at temperatures between $20^{\circ} \mathrm{C}$ and $68^{\circ} \mathrm{C}$ and are found mainly in Australia, Asia (Thailand, Taiwan and Hong Kong), the Gulf of Mexico and South America as described by the Centers for Disease Control [25]. These findings are consistent with the fact that most of our study patients with Vibrio infections were from the Chiayi Chang Gung Memorial Hospital study site located close to two local harbors. Vibrio spp. (usually $V$. vulnificus) is known to cause severe necrotizing wound infection, necrotizing fasciitis and sepsis $[23,26]$, resulting in a separate classification of Type 3 necrotizing fasciitis caused by marine Vibrio spp. [13,27]. Ingestion of $V$. vulnificus in the form of contaminated 
Table 6 Univariate logistic regression analysis of mortality related to multiple prognostic factors; $(N=323)$

\begin{tabular}{|c|c|c|c|c|}
\hline Variables & $\begin{array}{l}\text { Survival } \\
(n=271)\end{array}$ & $\begin{array}{l}\text { Death } \\
(n=52)\end{array}$ & OR $\left(95 \% \mathrm{Cl}^{3}\right)^{3}$ & $P$-value ${ }^{4}$ \\
\hline $\mathrm{Age}^{1}$, years & $57.4 \pm 15.5$ & $58.4 \pm 14.2$ & 1.00 (0.98 to 1.02$)$ & 0.607 \\
\hline Gender $^{2}$, males (\%) & $192(70.8)$ & $32(61.5)$ & 1.24 (0.64 to 2.41$)$ & 0.525 \\
\hline \multicolumn{5}{|l|}{ Pre-existing disease ${ }^{2}$} \\
\hline Fever, n (\%) & $85(31.4)$ & $18(34.6)$ & 1.16 (0.62 to 2.17$)$ & 0.645 \\
\hline Tachycardia & $126(46.5)$ & $26(50.0)$ & 1.05 (0.58 to 1.90$)$ & 0.872 \\
\hline Hypotension & $30(11.1)$ & $12(23.1)$ & 7.07 (3.48 to 14.37$)$ & $<.001^{*}$ \\
\hline Diabetes mellitus & $167(61.6)$ & $28(53.8)$ & 1.06 (0.58 to 1.95$)$ & 0.851 \\
\hline Heart disease & $37(13.7)$ & $6(11.5)$ & $3.52(1.72$ to 7.20$)$ & $0.001^{*}$ \\
\hline Peripheral vascular disease & $23(8.5)$ & $4(7.7)$ & 0.39 (0.09 to 1.71$)$ & 0.214 \\
\hline Renal disease & $49(18.1)$ & $11(21.2)$ & 1.59 (0.79 to 3.21$)$ & 0.196 \\
\hline Liver disease & $74(27.3)$ & $17(32.7)$ & 2.37 (1.28 to 4.37$)$ & $0.006^{*}$ \\
\hline Intravenous drug use & $14(5.2)$ & $1(1.9)$ & 0.36 (0.05 to 2.80) & 0.329 \\
\hline \multicolumn{5}{|l|}{ Clinical features ${ }^{2}$} \\
\hline Affected site (limbs) & $228(84.1)$ & $48(92.3)$ & 1.11 (0.47 to 2.64) & 0.808 \\
\hline Bullae formation & $109(40.2)$ & $23(44.2)$ & 1.18 (0.65 to 2.15$)$ & 0.590 \\
\hline Operation within 24 hours & $187(69.0)$ & $35(67.3)$ & $0.68(0.35$ to 1.26$)$ & 0.224 \\
\hline Amputation & $70(25.8)$ & $13(25.0)$ & 1.86 (0.99 to 3.50$)$ & 0.053 \\
\hline Debridement $>=3$ times & $157(57.9)$ & $11(21.2)$ & 0.19 (0.10 to 0.39$)$ & $<.001^{*}$ \\
\hline Multiple Etiologic agent in Wound culture ${ }^{2}, \mathrm{n}(\%)$ & $129(47.6)$ & $24(46.2)$ & 0.94 (0.52 to 1.71$)$ & 0.848 \\
\hline \multicolumn{5}{|l|}{ Gram positive organism in wound culture ${ }^{2}$} \\
\hline Streptococcus group A & $32(11.8)$ & $5(9.6)$ & $0.79(0.29$ to 2.14$)$ & 0.650 \\
\hline Streptococcus group B & $4(1.5)$ & $2(3.8)$ & 2.67 (0.48 to 14.97$)$ & 0.264 \\
\hline $\begin{array}{l}\text { Methicillin-susceptible } \\
\text { S. aureus }\end{array}$ & $39(14.4)$ & $3(5.8)$ & $0.36(0.11$ to 1.23$)$ & 0.103 \\
\hline Methicillin-resistant S. aureus & $47(17.3)$ & $7(13.5)$ & $0.74(0.31$ to 1.74$)$ & 0.493 \\
\hline \multicolumn{5}{|l|}{ Gram negative organism in wound culture ${ }^{2}$} \\
\hline Acinetobacter baumannii & $20(7.4)$ & $3(5.8)$ & 0.77 (0.22 to 2.69) & 0.680 \\
\hline Aeromonas spp. & $18(6.6)$ & $4(7.7)$ & 1.17 (0.38 to 3.61$)$ & 0.783 \\
\hline E.coli & $29(10.7)$ & $8(15.4)$ & 1.52 (0.65 to 3.54$)$ & 0.334 \\
\hline K.pneumoniae & $36(13.3)$ & $5(9.6)$ & 0.69 (0.26 to 1.86$)$ & 0.469 \\
\hline Proteus mirrabilis & $23(8.5)$ & $0(0)$ & - & - \\
\hline Pseudomonas spp. & $16(5.9)$ & $3(5.8)$ & 0.98 (0.27 to 3.48$)$ & 0.970 \\
\hline Vibrio spp. & $11(4.1)$ & $11(21.2)$ & 6.37 (2.58 to 15.57$)$ & $<.001^{*}$ \\
\hline Fungus in wound culture ${ }^{2}$ & $6(2.2)$ & $1(1.9)$ & $6.34(2.58$ to 15.57$)$ & $<.001^{*}$ \\
\hline Multiple Etiologic agent in blood culture ${ }^{2}, \mathrm{n}(\%)$ & $3(1.1)$ & $1(1.9)$ & 1.75 (0.18 to 17.17$)$ & 0.630 \\
\hline \multicolumn{5}{|l|}{ Gram positive organism in blood culture ${ }^{2}$} \\
\hline Streptococcus group A & $2(0.7)$ & $3(5.8)$ & 8.24 (1.34 to 50.56$)$ & $0.023^{*}$ \\
\hline Streptococcus group B & $1(0.4)$ & $1(1.9)$ & $5.29(0.33$ to 86.01$)$ & 0.241 \\
\hline $\begin{array}{l}\text { Methicillin-susceptible } \\
\text { S. aureus }\end{array}$ & $10(3.7)$ & $0(0)$ & - & - \\
\hline Methicillin-resistant S. aureus & $5(1.8)$ & $2(3.8)$ & 2.13 (0.40 to 11.27$)$ & 0.375 \\
\hline \multicolumn{5}{|l|}{ Gram negative organism in blood culture ${ }^{2}$} \\
\hline Acinetobacter baumannii & $0(0)$ & $2(3.8)$ & - & - \\
\hline Aeromonas spp. & $3(1.1)$ & $3(5.8)$ & 5.47 (1.07 to 27.89$)$ & $0.041^{*}$ \\
\hline E.coli & $3(1.1)$ & $2(3.8)$ & 3.57 (0.58 to 21.93$)$ & 0.169 \\
\hline Proteus mirrabilis & $0(0)$ & $1(1.9)$ & - & - \\
\hline Vibrio spp. & $11(4.1)$ & $9(17.3)$ & 4.95 (1.94 to 12.64$)$ & $0.001^{*}$ \\
\hline
\end{tabular}

1,2 Data were summarized as mean $\pm \mathrm{SD}$, and $\mathrm{n}(\%)$ for continuous, and categorical variables for subjects by survival status, respectively.

${ }^{3}$ OR: $(95 \% \mathrm{Cl})$, Odds ratio with $95 \%$ confidence interval were derived through univariate logistic regression model.

${ }^{4} P$-values from univariate logistic regression analysis, were used to identify the significance of respective odds ratio $(\mathrm{OR}) * P<0.05$. 
Table 7 Multivariate logistic regression analysis of mortality related to multiple prognostic factors; $(\mathrm{N}=323)$

\begin{tabular}{|c|c|c|}
\hline Variables & OR $(95 \% \mathrm{Cl})^{1}$ & $P$-value ${ }^{2}$ \\
\hline Age, years & 0.99 (0.97 to 1.02$)$ & 0.607 \\
\hline Gender, males (\%) & $1.16(0.50$ to 2.67$)$ & 0.731 \\
\hline \multicolumn{3}{|l|}{ Pre-existing disease } \\
\hline Hypotension & $5.68(2.21$ to 14.59$)$ & $<0.001^{*}$ \\
\hline Heart disease & 2.89 (1.09 to 7.62$)$ & $0.033^{*}$ \\
\hline Liver disease & $2.54(1.10$ to 5.87$)$ & $0.029^{*}$ \\
\hline \multicolumn{3}{|l|}{ Clinical features } \\
\hline Operation within 24 hours & $0.59(0.27$ to 1.29$)$ & 0.182 \\
\hline Debridement $>=3$ times & 0.19 (0.08 to 0.43$)$ & $<.001^{*}$ \\
\hline \multicolumn{3}{|c|}{ Gram negative organism in wound culture } \\
\hline Vibrio spp. & $5.60(1.82$ to 17.25$)$ & $0.003^{*}$ \\
\hline Fungus in wound culture & $0.52(0.04$ to 7.54$)$ & 0.632 \\
\hline \multicolumn{3}{|c|}{ Gram positive organism in blood culture } \\
\hline Streptococcus group A & $15.93(1.95$ to 130.34$)$ & $0.010^{*}$ \\
\hline \multicolumn{3}{|c|}{ Gram negative organism in blood culture } \\
\hline Aeromonas spp. & 3.50 (0.46 to 26.76$)$ & 0.227 \\
\hline Vibrio spp. & 1.19 (0.29 to 4.92$)$ & 0.806 \\
\hline
\end{tabular}

${ }^{1}$ Odds ratio with $95 \%$ confidence interval (OR: $(95 \% \mathrm{Cl})$ were derived through multivariate logistic regression model.

${ }^{2} P$-values from multivariate logistic regression analysis, were used to identify the significance of respective OR. ${ }^{*} P<0.05$.

seafood, such as raw fish, raw oysters or from open wounds that have been exposed to seawater, can lead to bloodstream infection and NF, especially in immunocompromised patients, with a mortality rate of about 50\% [23]. Our results were consistent with these data and showed that all our study patients with Vibrio infection (21 cases) either had a history of an open wound which was exposed to seawater or a history of eating raw seafood. Twenty of these patients developed bloodstream infections.

Clostridial infections, although rare, are fulminant and fatal infections resulting in myonecrosis and are an independent predictor for limb loss and mortality [11]. Our results were consistent with these data and showed higher mortality rates in patients infected with Clostridium spp. However, the number of Clostridial infections in our study was smaller (five cases) than previously published reports. Clostridial infections were previously shown to be significantly associated with intravenous drug use. The small number of NF patients with a history of intravenous drug use in our study ( 15 cases), could explain the smaller number of clostridial infections in our study population.

Bloodstream infection is associated with increased morbidity, duration of hospital stay and mortality $[28,29]$ and was previously shown to be a significant risk factor for mortality in NF patients $[6,12,30,31]$. Our data showed that patients with positive blood stream infections had a 2.26-fold higher incidence of death, while there was no significant association between positive wound cultures and mortality. Despite recent advances in medical care, invasive Group A Streptococcus-induced NF patients have high mortality rates (ranging from $18 \%$ to $34 \%$ ) [32,33]. The reported rates of bacteremia with Group A streptococci range from 46 to $85 \%$ [34-36]. Our results showed that patients with Group A Streptococcus in blood cultures had significantly higher mortality rates when compared with patients negative for Group A Streptococcus. This is possibly due to the presence of toxic shock/myositis, reflecting the amount of circulating exotoxin. Our multivariate analysis showed that increased mortality was associated with the presence of Vibrio spp. in wound cultures and Streptococcus Group A in blood cultures.

Independent predictors of mortality in NF patients, such as age, gender, existence of comorbidities or serum lactate and sodium levels at the time of admission, have not proved definitive $[11,37]$. However, early surgical intervention is associated with decreased mortality rates $[2,38,39]$. A study of mortality in NF patients showed a significantly longer average time ( 90 hours) from admission to surgery among non-survivors when compared with survivors (25 hours) [2]. We previously showed that a prolonged ED boarding stay was associated with increased mortality, while early operation (within 24 hours of admission) was

Table 8 Univariate analysis of mortality related to blood stream infection and positive wound cultures

\begin{tabular}{lllll}
\hline Variables & Survival $(\boldsymbol{n}=\mathbf{2 7 1})$ & Death $(\boldsymbol{n}=\mathbf{5 2})$ & OR $(\mathbf{9 5 \%} \text { Cl. })^{\mathbf{3}}$, & $\boldsymbol{P}_{\text {-value }}{ }^{\mathbf{4}}$ \\
\hline Positive blood stream infection & $48(17.7)$ & $17(32.7)$ & $2.26(1.17$ to 4.36$)$ & 0.015 \\
Wound culture positive & $235(86.7)$ & $44(84.6)$ & $0.84(0.37$ to 1.93) & 0.686 \\
\hline
\end{tabular}


associated with decreased mortality [40]. Our present data on patient mortality were consistent with some previous studies $[1,11,37]$ but were significantly lower than other previously reported data [2-6]. Most of the NF patients in our study (68.7\%) received surgical intervention within 24 hours of admission. This may explain 1) the lack of a significant association between the time of surgical intervention and outcomes and 2) the lower mortality seen in our patients.

The retrospective nature of our study is one of the major limitations. We are aware that reviewed medical charts may not contain all variables of interest and may have inconsistent descriptions. In order to overcome these issues, we had all the charts reviewed by two principal investigators. Furthermore, we used standardized chart review procedures to ensure the quality of data collection and to increase the validity and reliability of the data collected. Despite these limitations, ours is the first multi-institutional study, assessing the microbiologic aspects of necrotizing fasciitis in a large number of NF patients and identifying the association between the microbiological characteristics of wound and blood cultures and mortality. These data are key in designing earlier treatment options and more efficient prognostic strategies for the management of NF.

\section{Conclusions}

We characterized the causative microorganisms in a cohort of 323 necrotizing fasciitis patients and determined the relationship between mortality and the causative pathogens of a wound or blood stream infection in these patients.

We showed that hypotension, heart disease, liver disease, the presence of Vibrio spp. in wound cultures, presence of fungus in wound cultures and presence of Streptococcus group A, Aeromonas spp. or Vibrio spp. in blood cultures were associated with a higher risk of mortality. Debridement $>=3$ times and early surgical intervention were associated with a lower risk of mortality.

\section{Key messages}

- NF is caused by a number of different pathogens.

- Hypotension, heart disease and liver disease were associated with higher mortality in NF patients.

- Presence of Vibrio spp in wound cultures, and Streptococcus Group A in blood cultures were associated with a high risk of mortality.

- Patients with positive blood stream infections had a 2.26-fold higher incidence of death, while there was no significant association between positive wound cultures and mortality.

- Debridement $>=3$ times had a lower risk of mortality.

\section{Abbreviations}

BSI: bloodstream infection; ED: emergency department; LRINEC: laboratory risk indicator for necrotizing fasciitis; MRSA: methicillin-resistant

Staphylococcus aureus; MSSA: methicillin-susceptible S. aureus; NF: necrotizing fasciitis.

\section{Acknowledgements}

We thank Professor Tsung Han Yang for providing valuable information about process of wound and blood culture in our clinical laboratory. This study does not receive financial support.

\section{Author details}

${ }^{1}$ Department of Emergency Medicine, Chang Gung Memorial Hospital, No.6, W. Sec., Jiapu Rd., Puzih City, Chiayi County 613, Taiwan. ${ }^{2}$ Division of Infectious Diseases, Department of Internal Medicine, Chang Gung Memorial Hospital, No.6, W. Sec., Jiapu Rd., Puzih City, Chiayi County 613, Taiwan. ${ }^{3}$ Chang Gung University College of Medicine, No.5, Fusing St., Gueishan Township, Taoyuan County 333, Taiwan. ${ }^{4}$ Department of Occupation Medicine, Chang-Gung Memorial Hospital, Keelung Branch, No. 222, Maijin Rd., Keelung, Taiwan.

\section{Authors' contributions}

WCL and YCH conceived the study, designed the method, supervised the conduct of the data collection, undertook recruitment of participating centers and patients and managed the data, including quality control. ICC conceived the study, designed the method, supervised the conduct of the data collection, undertook recruitment of participating centers and patients and managed the data, including quality control, provided statistical advice on study design and analyzed the data and drafted the manuscript. SSS conceived the study, designed the method, supervised the conduct of the data collection, provided statistical advice on study design and analyzed the data. CTH supervised the conduct of the data collection, chaired the data oversight committee and drafted the manuscript. WCF undertook recruitment of participating centers and patients and managed the data, including quality control and all authors contributed substantially to its revision. ICC and CTH take responsibility for the paper as a whole. All the authors have read and approved the final version of the manuscript.

\section{Competing interests}

The authors declare that they have no competing interests.

Received: 6 January 2011 Revised: 27 May 2011

Accepted: 21 June 2011 Published: 21 June 2011

\section{References}

1. Tillou A, St Hill CR, Brown C, Velmahos G: Necrotizing soft tissue infections: improved outcomes with modern care. Am Surg 2004, 70:841-844.

2. McHenry CR, Piotrowski JJ, Petrinic D, Malangoni MA: Determinants of mortality for necrotizing soft-tissue infections. Ann Surg 1995, 221:558-563, discussion 563-555.

3. Elliott DC, Kufera JA, Myers RA: Necrotizing soft tissue infections. Risk factors for mortality and strategies for management. Ann Surg 1996, 224:672-683.

4. Faucher LD, Morris SE, Edelman LS, Saffle JR: Burn center management of necrotizing soft-tissue surgical infections in unburned patients. Am J Surg 2001, 182:563-569.

5. Singh G, Sinha SK, Adhikary S, Babu KS, Ray P, Khanna SK: Necrotising infections of soft tissues-a clinical profile. Eur J Surg 2002, 168:366-371.

6. Childers BJ, Potyondy LD, Nachreiner R, Rogers FR, Childers ER, Oberg KC, Hendricks DL, Hardesty RA: Necrotizing fasciitis: a fourteen-year retrospective study of 163 consecutive patients. Am Surg 2002, 68:109-116.

7. Wilkinson D, Doolette D: Hyperbaric oxygen treatment and survival from necrotizing soft tissue infection. Arch Surg 2004, 139:1339-1345.

8. Law D, Dhingra KR: Necrotizing fasciitis of the paraspinous muscles. West J Emerg Med 11:68-70.

9. Su YC, Chen HW, Hong YC, Chen $C T$, Hsiao $C T$, Chen IC: Laboratory risk indicator for necrotizing fasciitis score and the outcomes. ANZ J Surg 2008, 78:968-972. 
10. Ellis Simonsen SM, van Orman ER, Hatch BE, Jones SS, Gren LH, Hegmann KT, Lyon JL: Cellulitis incidence in a defined population. Epidemiol Infect 2006, 134:293-299.

11. Anaya DA, McMahon K, Nathens AB, Sullivan SR, Foy H, Bulger E: Predictors of mortality and limb loss in necrotizing soft tissue infections. Arch Surg 2005, 140:151-157, discussion 158.

12. Elliott $D$, Kufera JA, Myers RA: The microbiology of necrotizing soft tissue infections. Am J Surg 2000, 179:361-366.

13. Fontes RA Jr, Ogilvie CM, Miclau T: Necrotizing soft-tissue infections. J Am Acad Orthop Surg 2000, 8:151-158.

14. Giuliano A, Lewis F Jr, Hadley K, Blaisdell FW: Bacteriology of necrotizing fasciitis. Am J Surg 1977, 134:52-57.

15. Morgan MS: Diagnosis and management of necrotising fasciitis: a multiparametric approach. J Hosp Infect 2010, 75:249-257.

16. Taviloglu K, Yanar H: Necrotizing fasciitis: strategies for diagnosis and management. World J Emerg Surg 2007, 2:19.

17. Kim HJ, Kim DH, Ko DH: Coagulase-positive staphylococcal necrotizing fasciitis subsequent to shoulder sprain in a healthy woman. Clin Orthop Surg 2010, 2:256-259.

18. Puvanendran R, Huey JC, Pasupathy S: Necrotizing fasciitis. Can Fam Physician 2009, 55:981-987.

19. Taviloglu K, Cabioglu N, Cagatay A, Yanar H, Ertekin C, Baspinar I, Ozsut H, Guloglu R: Idiopathic necrotizing fasciitis: risk factors and strategies for management. Am Surg 2005, 71:315-320.

20. Hsiao CT, Weng HH, Yuan YD, Chen $C T$, Chen IC: Predictors of mortality in patients with necrotizing fasciitis. Am J Emerg Med 2008, 26:170-175.

21. Bone RC, Balk RA, Cerra FB, Dellinger RP, Fein AM, Knaus WA, Schein RM, Sibbald WJ: Definitions for sepsis and organ failure and guidelines for the use of innovative therapies in sepsis. The ACCP/SCCM Consensus Conference Committee. American College of Chest Physicians/Society of Critical Care Medicine. Chest 1992, 101:1644-1655.

22. Levy MM, Fink MP, Marshall JC, Abraham E, Angus D, Cook D, Cohen J, Opal SM, Vincent JL, Ramsay G: 2001 SCCM/ESICM/ACCP/ATS/SIS International Sepsis Definitions Conference. Crit Care Med 2003, 31:1250-1256.

23. Kuo YL, Shieh SJ, Chiu HY, Lee JW: Necrotizing fasciitis caused by Vibrio vulnificus: epidemiology, clinical findings, treatment and prevention. Eur J Clin Microbiol Infect Dis 2007, 26:785-792.

24. Angoules AG, Kontakis G, Drakoulakis E, Vrentzos G, Granick MS, Giannoudis PV: Necrotising fasciitis of upper and lower limb: a systematic review. Injury 2007, 38(Suppl 5):S19-26.

25. CDC - Vibrio vulnificus: General Information - NCZVED. [http://www.cdc. gov/nczved/divisions/dfbmd/diseases/vibriov/]

26. Tsai YH, Hsu RW, Huang TJ, Hsu WH, Huang KC, Li YY, Peng KT: Necrotizing soft-tissue infections and sepsis caused by Vibrio vulnificus compared with those caused by Aeromonas species. J Bone Joint Surg Am 2007, 89:631-636.

27. Salcido RS: Necrotizing fasciitis: reviewing the causes and treatment strategies. Adv Skin Wound Care 2007, 20:288-293, quiz 294-285.

28. Warren DK, Zack JE, Elward AM, Cox MJ, Fraser VJ: Nosocomial primary bloodstream infections in intensive care unit patients in a nonteaching community medical center: a 21-month prospective study. Clin Infect Dis 2001, 33:1329-1335.

29. Orsi GB, Di Stefano L, Noah N: Hospital-acquired, laboratory-confirmed bloodstream infection: increased hospital stay and direct costs. Infect Control Hosp Epidemiol 2002, 23:190-197.

30. Ben-Abraham R, Keller N, Vered R, Harel R, Barzilay Z, Paret G: Invasive group A streptococcal infections in a large tertiary center: epidemiology, characteristics and outcome. Infection 2002, 30:81-85.

31. Kaul R, McGeer A, Low DE, Green K, Schwartz B: Population-based surveillance for group A streptococcal necrotizing fasciitis: Clinical features, prognostic indicators, and microbiologic analysis of seventyseven cases. Ontario Group A Streptococcal Study. Am J Med 1997. 103:18-24.

32. Kaul R, McGeer A, Norrby-Teglund A, Kotb M, Schwartz B, O'Rourke K, Talbot J, Low DE: Intravenous immunoglobulin therapy for streptococcal toxic shock syndrome-a comparative observational study. The Canadian Streptococcal Study Group. Clin Infect Dis 1999, 28:800-807.

33. Hollm-Delgado MG, Allard R, Pilon PA: Invasive group A streptococcal infections, clinical manifestations and their predictors, Montreal, 19952001. Emerg Infect Dis 2005, 11:77-82.
34. Mehta S, McGeer A, Low DE, Hallett D, Bowman DJ, Grossman SL, Stewart TE: Morbidity and mortality of patients with invasive group A streptococcal infections admitted to the ICU. Chest 2006, 130:1679-1686.

35. Sharkawy A, Low DE, Saginur R, Gregson D, Schwartz B, Jessamine $P$, Green K, McGeer A: Severe group a streptococcal soft-tissue infections in Ontario: 1992-1996. Clin Infect Dis 2002, 34:454-460.

36. Davies HD, McGeer A, Schwartz B, Green K, Cann D, Simor AE, Low DE: Invasive group A streptococcal infections in Ontario, Canada. Ontario Group A Streptococcal Study Group. N Engl J Med 1996, 335:547-554.

37. Yaghoubian A, de Virgilio C, Dauphine C, Lewis RJ, Lin M: Use of admission serum lactate and sodium levels to predict mortality in necrotizing softtissue infections. Arch Surg 2007, 142:840-846, discussion 844-846.

38. Wong $\mathrm{CH}$, Chang HC, Pasupathy S, Khin LW, Tan JL, Low CO: Necrotizing fasciitis: clinical presentation, microbiology, and determinants of mortality. J Bone Joint Surg Am 2003, 85-A:1454-1460.

39. Bilton BD, Zibari GB, McMillan RW, Aultman DF, Dunn G, McDonald JC Aggressive surgical management of necrotizing fasciitis serves to decrease mortality: a retrospective study. Am Surg 1998, 64:397-400, discussion 400-391.

40. Hong YC, Chou MH, Liu EH, Hsiao CT, Kuan JT, Lin JC, Chen IC: The effect of prolonged ED stay on outcome in patients with necrotizing fasciitis. Am J Emerg Med 2009, 27:385-390.

doi: $10.1186 /$ cc10278

Cite this article as: Chen et al:: The microbiological profile and presence of bloodstream infection influence mortality rates in necrotizing fasciitis. Critical Care 2011 15:R152.

\section{Submit your next manuscript to BioMed Central and take full advantage of:}

- Convenient online submission

- Thorough peer review

- No space constraints or color figure charges

- Immediate publication on acceptance

- Inclusion in PubMed, CAS, Scopus and Google Scholar

- Research which is freely available for redistribution

Submit your manuscript at www.biomedcentral.com/submit
Biomed Central 